\title{
Effectiveness of Vaccination Strategies for Infectious Diseases According to Human Contact Networks
}

\author{
Fumihiko Takeuchi and Kenji Yamamoto \\ Department of Infection Control Science, Juntendo University, 113-8421, Tokyo, Japan \\ Research Institute, International Medical Center of Japan, 162-8655, Tokyo, Japan \\ fumihiko@takeuchi.name
}

\begin{abstract}
A 'contact network' modeling infection transmission comprises of nodes (or individuals) that are linked when they are in contact that possibly transmits an infection. We here studied infection transmission on contact networks of various degree distributions-scale-free, exponential and constant-under SIRV model assuming susceptible, infected, removed and vaccinated statuses of nodes. Aiming for infectious disease containment within the very early stage of spreading, we computed the minimum transmissibility at which an infectious disease epidemic begins to emerge, and its change according to mass preventive and ring post-outbreak vaccination. In the most degree-heterogeneous scale-free network, the 'super-spreading' by the hubs, or high-degree nodes, allowed epidemics even for low transmissibility. In compensation, vaccination was much more efficient for the scale-free network. We also found that basic reproductive number $R_{0}$ defines a measurement of epidemic emergence universally applicable to networks of various degree distributions. These results are significant for public health design.
\end{abstract}

\section{Introduction}

A 'contact network' modeling infection transmission comprises of nodes (or individuals) that are linked when they are in contact that possibly transmits an infection. Here, the magnitude of the spreading of infection is determined not solely by the infectiousness of the pathogen but also by the structure of the contact network. In particular, a major factor is the distribution of each node's 'degree,' which is the number of nodes linked to it. If all nodes have the same degree and the links between nodes are random, there exists a threshold value in 'transmissibility,' the probability that an infected node transmits the infection to a susceptible node in contact, less than which an outbreak immediately extinguishes as an endemic [1]. On the other hand, if there are a significant number of high-degree nodes, or hubs, such nodes can become super-spreaders, and allow an outbreak even under weak transmissibility. In fact, in scale-free networks, which only have a power decrease in the number of high-degree nodes, there remains a marginal number of stationary infected nodes under SIS model (nodes transit between susceptible and infected), even for pathogens of infinitely small transmissibility [2]. This phenomenon is typically observed as computer virus infections in the Internet.

Vulnerability to infection attributable to the heterogeneous degree distribution is applicable to contact networks of infectious diseases as well, thus can become a 
concern for public health. Although our knowledge on the contact network of infectious diseases is limited, social networks can give some insight. Sexual contacts that follow the scale-free degree distribution [3] must be those with largest degreeheterogeneity, whereas friendship relations that follow Gaussian distribution [4] must include much less number of hubs. Studies on such infections and their containment are important both for existing diseases such as AIDS or SARS or for those introduced deliberately by bioterrorism such as smallpox.

The primary measure for containing infection is vaccination, either preventive or post-outbreak. The epidemic mentioned above under SIS model in scale-free networks cannot be stopped by preventive mass vaccination of randomly selected nodes even of a large proportion, but can be halted by prioritized vaccination of hub nodes $[5,6]$. However, for vaccination in case of infectious diseases in human, the latter hub vaccination is difficult to implement, because the contact network is not apparent and potential hubs are not evident. On the other hand, among post-outbreak vaccination strategies, the one important in practice is the ring vaccination, in which the susceptible individuals in contact with an infected individual are vaccinated. Yet, there has been no study evaluating the effectiveness of ring vaccination or its combination with preventive mass vaccination, the two practical containment strategies. Moreover, for the study of infectious diseases in human a modeling more realistic than the SIS is necessary.

Thus, we here studied infection transmission on contact networks of various degree distributions under SIRV model assuming not only susceptible and infected nodes, but also those removed (by death or acquiring immunity) and vaccinated, and evaluated the effectiveness of mass preventive and ring post-outbreak vaccinations.

\section{Results}

Aiming for infectious disease containment within the very early stage of spreading, we studied the minimum transmissibility at which an infectious disease epidemic begins to emerge, and its change according to contact networks or vaccination strategies. We generated random contact networks comprising of $n=100,000$ nodes having average degree $\langle k\rangle=10$ with high-degree nodes decreasing by power (scale-free network), exponentially (exponential network), or with all nodes having degree $\langle k\rangle$ thus with no high-degree nodes (constant network) (Figure 1). Under no vaccination, the scale-free network that has the largest heterogeneity of degree allowed epidemic emergence even for transmissibility $T=0.032$, which was followed by $T=0.087$ for the less heterogeneous exponential network, and then by $T=0.111$ for the homogeneous constant network (Figure 2, Table 1). This exhibits the vulnerability of degreeheterogeneous contact networks to infection.

Although the transmissibilities causing epidemic emergence were different among the networks, the corresponding values of basic reproductive number $R_{0}$ were consistenly around one (Table 1 ). This number $R_{0}$ is defined as the expected number of secondary infections among nodes in contact with a primary infected node and is evaluated as $R_{0}=\left(\left\langle k^{2}\right\rangle /\langle k\rangle-1\right) T$ [7]. Whereas the transmissibility $T$ basically defines the pathogen's biological strength for transmission, $R_{0}$ indicates the strength of spreading in a specific contact network, and reflects the degree distribution of the 
network. In particular, the value $R_{0}$ can become large even under a small $T$, when the degree distrubution is heterogeneous and $\left\langle k^{2}\right\rangle\langle\langle k\rangle$ is large. Furthermore, we observed the epidemic emergence to occur around $R_{0}=1$ independently of the number of nodes (see the case $n=10,000$ in Table 1) or the average degree (see the case $\langle k\rangle=100$ ). These results indicate that basic reproductive number serves as a faithful indicator for the emergence of epidemic independently of the degree distribution.

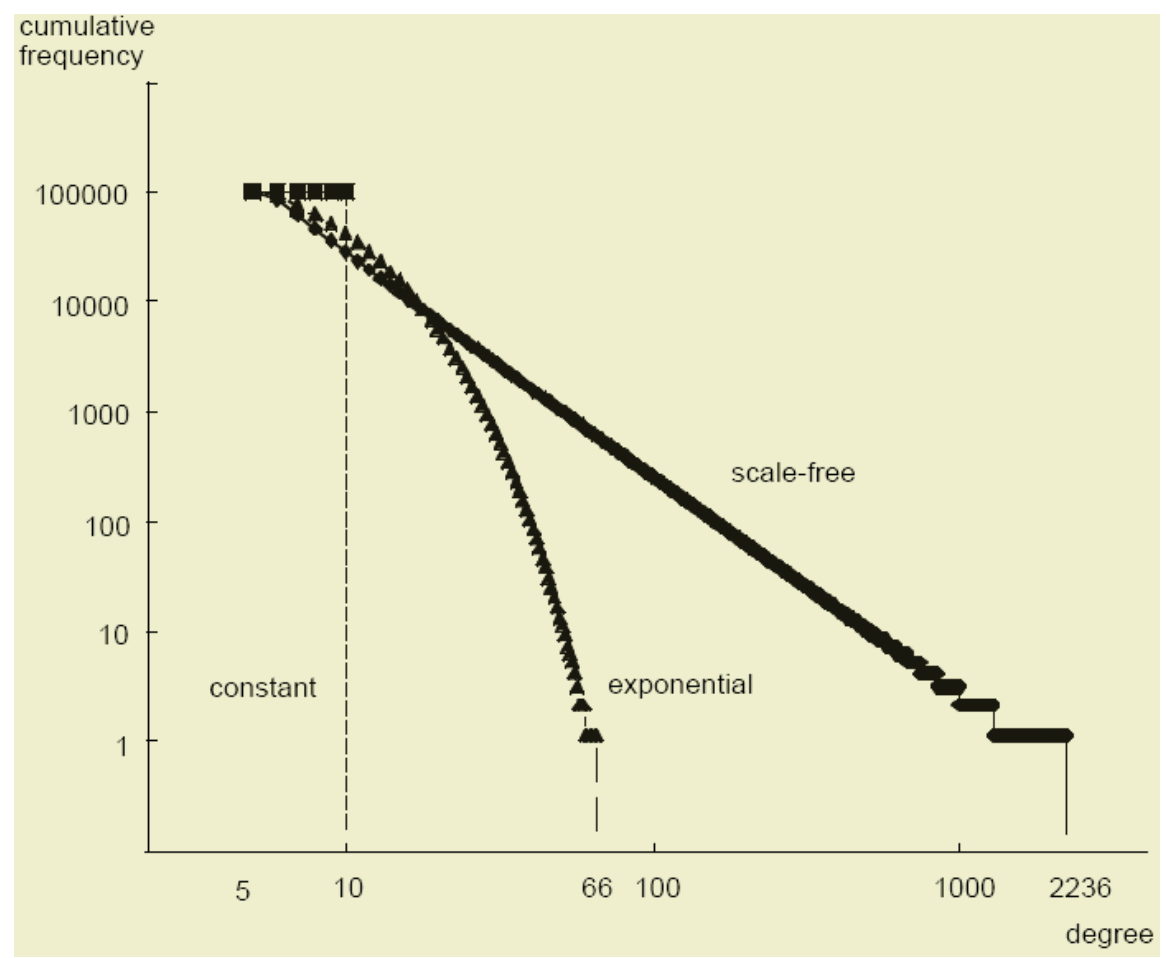

Fig. 1. Cumulative frequency of nodes according to degree for scale-free, exponential and constant networks of $n=100,000$ nodes with average degree $\langle k\rangle=10$

Compensating the vulnerability of scale-free networks to weak transmissibility, vaccination worked much effectively for such networks compared to the exponential and constant networks. We simulated for various ratio $u$ of randomly selected nodes preventively vaccinated (mass preventive vaccination) and ratio $v$ of susceptible status nodes in contact with an infected node to be vaccinated (ring vaccination). In exponential or constant networks, the transmissibility necessary to cause emergence of epidmic was enlarged by the factor of $1 /[(1-u) \cdots(1-v)]$ compared to the case without vaccination (Table 1). This can be explained as vaccinations decreasing the number of (susceptible) nodes around an infected node approximately by the factor of $(1-u) \cdots(1-v)$. In scale-free networks, on the other hand, the transmissibility necessary for epidemic was observed to increase by a much larger factor of $16^{u+v}$ (Table 1). For example, a pathogen of transmissibility $T=0.018$ could emerge an epidemic under no vaccination, whereas the value arose to $T=0.123$ under $u+v=0.5$. 


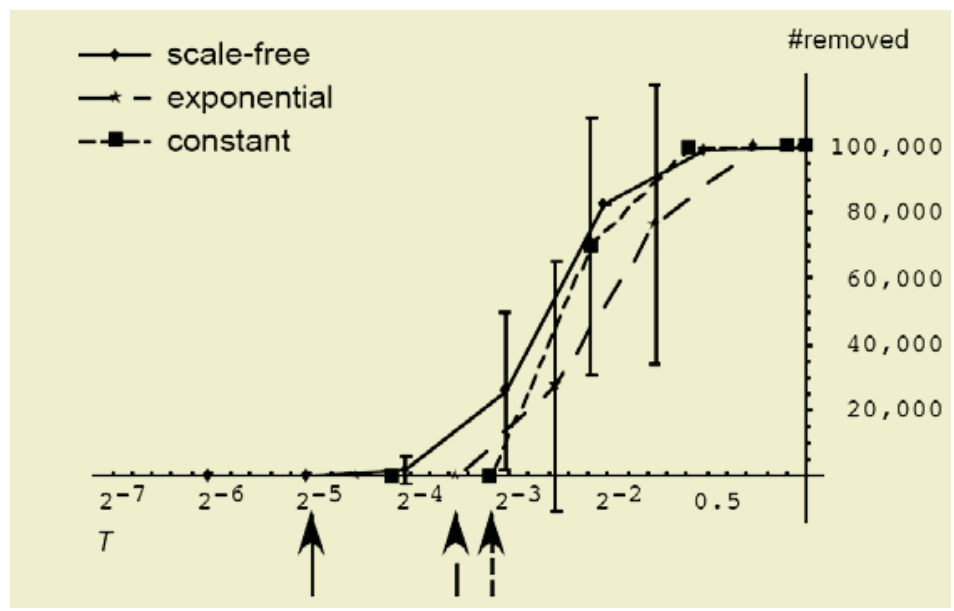

Fig. 2. The number of removed nodes after infection simulation for scale-free, exponential and constant networks of $n=100,000$ nodes with average degree $\langle k\rangle=10$ under various transmissibility, and without vaccination. Horizontal axis indicates the transmissibility $T$, and vertical axis indicates the final number of removed nodes. Data points for the scale-free network are diamonds connected by lines, those for the exponential network are stars connected by sparsely dashed lines, and those for the constant network are squares connected by densely dashed lines. Standard deviation of five experiments is indicated by a vertical error bar centered at the mean point. The transmissibility (interpolated in log scale) for which 100 nodes are removed was defined as the epidemic emerging transmissbility, and the values for the three networks are indicated by arrowed vertical lines of the same type as those connecting data points

Table 1. Transmissibility $T$ and basic reproductive number $R_{0}$ causing emergence of epidemic for various contact networks

\begin{tabular}{|c|c|c|c|c|c|c|c|c|c|c|c|}
\hline \multirow[b]{2}{*}{$n$} & \multirow[b]{2}{*}{$\langle k\rangle$} & \multirow[b]{2}{*}{ initial infection } & \multicolumn{3}{|l|}{ vaccination } & \multicolumn{2}{|c|}{ scale-free } & \multicolumn{2}{|c|}{ exponential } & \multicolumn{2}{|c|}{ constant } \\
\hline & & & $\begin{array}{l}\text { mass } \\
\text { preventive }\end{array}$ & ring & & $T$ & $R_{0}$ & $T$ & $R_{0}$ & $T$ & $R_{0}$ \\
\hline 100,000 & & random & $0 \%$ & & $0 \%$ & 0.032 & 1.04 & 0.087 & 1.00 & 0.111 & 1.00 \\
\hline 100,000 & & random & $0 \%$ & & $25 \%$ & 0.064 & 2.08 & 0.088 & 1.01 & 0.111 & 1.00 \\
\hline 100,000 & & random & $0 \%$ & & $50 \%$ & 0.123 & 4.03 & 0.174 & 2.01 & 0.222 & 2.00 \\
\hline 100,000 & & random & $0 \%$ & & $75 \%$ & 0.251 & 8.21 & 0.350 & 4.03 & 0.446 & 4.01 \\
\hline 100,000 & & random & $25 \%$ & & $0 \%$ & 0.062 & 2.03 & 0.088 & 1.01 & 0.111 & 1.00 \\
\hline 100,000 & 10 & random & $25 \%$ & & $25 \%$ & 0.123 & 4.04 & 0.095 & 1.09 & 0.115 & 1.04 \\
\hline 100,000 & & random & $25 \%$ & & $50 \%$ & 0.128 & 4.17 & 0.176 & 2.03 & 0.224 & 2.01 \\
\hline 100,000 & 10 & random & $25 \%$ & & $75 \%$ & 0.492 & 16.09 & 0.353 & 4.07 & 0.447 & 4.02 \\
\hline 100,000 & 10 & random & $50 \%$ & & $0 \%$ & 0.123 & 4.02 & 0.174 & 2.01 & 0.223 & 2.00 \\
\hline 100,000 & 10 & random & $50 \%$ & & $25 \%$ & 0.246 & 8.03 & 0.175 & 2.02 & 0.223 & 2.01 \\
\hline 100,000 & & random & $50 \%$ & & $50 \%$ & 0.254 & 8.32 & 0.349 & 4.02 & 0.445 & 4.00 \\
\hline 100,000 & & random & $50 \%$ & & $75 \%$ & 0.499 & 16.33 & 0.700 & 8.06 & 0.891 & 8.02 \\
\hline 100,000 & 10 & random & $75 \%$ & & $0 \%$ & 0.248 & 8.12 & 0.349 & 4.02 & 0.446 & 4.01 \\
\hline 100,000 & 10 & random & $75 \%$ & & $25 \%$ & 0.263 & 8.60 & 0.353 & 4.06 & 0.447 & 4.02 \\
\hline 100,000 & 10 & random & $75 \%$ & & $50 \%$ & 0.354 & 11.58 & 0.699 & 8.05 & 0.896 & 8.06 \\
\hline 100,000 & & random & $75 \%$ & & $75 \%$ & 0.761 & 24.90 & - & - & - & - \\
\hline 10,000 & & random & $0 \%$ & & $0 \%$ & 0.075 & 2.03 & 0.088 & 1.02 & 0.112 & 1.01 \\
\hline 100,000 & 100 & random & $0 \%$ & & $0 \%$ & 0.003 & 1.04 & 0.008 & 1.00 & 0.012 & 1.18 \\
\hline 100,000 & 10 & hub & $0 \%$ & & $0 \%$ & 0.018 & 0.58 & 0.057 & 0.66 & 0.111 & 1.00 \\
\hline
\end{tabular}

Dashes indicate absence of epidemic (even under $T=1$ ).

For each contact network, $R_{0}$ is calculated as $R_{0}=\left(\left\langle k^{2}\right\rangle|<k\rangle-1\right) T$ (see text). 
Finally, we evaluated how the choice of initially infected node affects epidemics. An infection transmits faster when a high-degree node is infected initially (say by hub targeted attack). However, even in the extreme case when the largest-degree node was initially infected, the transmissibility causing epidemic emergence became smaller only by the factor of 0.56 for scale-free network and 0.66 for the exponential, compared to the case when an initially infected node was selected randomly (Table 1).

\section{Discussion}

The 'super-spreading' by the hubs in the scale-free networks had significant effect of allowing epidemics even for low transmissibility. The demonstrated vulnerability of degree-heterogeneous contact networks under the SIRV model was in parallel to their vulnerability under SIS model in which infected nodes were stationarily reserved even for pathogens of weak infectiousness [2]. In compensation, vaccination turned out to be much more efficient for the scale-free network thus still enabling infectious disease containment in the very early stage of epidemics. In addition, the consistent measurement of epidemic emergence by basic reproductive number $R_{0}$ among the three types of networks indicated the appropriateness of the evaluation of infectiousness by $R_{0}$, which takes the degree heterogeneity into account. Our results could explain super-spreading caused simply by the structure of contact networks, but other factors such as pathogens changing transmissbility by recombination within a host, also can give accounts for super-spreaders.

\section{Methods}

\subsection{Generating Contact Networks}

We generated random contact networks of either $n=10,000$ or 100,000 nodes with average degree either $\langle k\rangle=10$ or 100 for three types of degree distributions in order to adjust the amount of hub nodes. In the scale-free degree distribution, the number of high-degree nodes decreased only by the power: the proportion of degree $k$ nodes among all nodes was set to be $p(k)=\left(m^{2} k^{-3}\right) / 2$ for $k \geq m / 2$, and $p(k)=0$ otherwise. In the exponential degree distribution, the high-degree nodes decreased exponentially: $p(k)=(2 e \exp (-2 k / m)) / m$ again for $k \geq m / 2$, and $p(k)=0$ otherwise. As for the other extremity, we tested the constant degree case, where all nodes had degree $m$, and there were no hubs at all. For each case, a contact network was generated by random connection of edges: firstly nodes with various degrees according to the distribution were listed, then 'untied links' emanating from each node by the number of its degree were generated, and finally the 'untied links' were connected randomly. The scalefree network had the largest number of high-degree nodes, the exponential network was in the medium, and the constant network had no such hubs (Figure 1). The mean squared degree $\left\langle k^{2}\right\rangle=\Sigma_{k} k^{2} p(k)$ of the generated scale-free network was 279.5 for $(n=10,000, m=10), 336.9$ for $(n=100,000, m=10)$ and 33,701.1 for $(n=100,000$, $m=100)$, that of the exponential network was 125.0 for $(n=10,000, m=10), 125.1$ for $(n=100,000, m=10)$ and $12,499.1$ for $(n=100,000, m=100)$, and that of the constant network was 100 for $(m=10)$ and 10,000 for $(m=100)$. 


\subsection{SIRV Model}

The nodes in our simulation had four possible statuses: susceptible, infected, removed or vaccinated. The simulation proceeds stepwise. In each step, a portion of 'susceptible' nodes that are vaccinated change to 'vaccinated.' Then, each 'infected' node transmits the infection to a 'susceptible' node in contact, by converting its status to 'infected' (in the next step) with probability $T$, the transmissibility. Meanwhile, all 'infected' nodes are changed to 'removed' in the next step. (The infected period is one step, which corresponds to various periods in reality depending on infectious diseases.) Thus, nodes in status 'removed' or 'vaccinated' do not change their status further. The simulation becomes stable and terminates when there are no more nodes in status 'infected.'

\subsection{Simulation of Infection and Vaccination}

For various contact networks and values of transmissibility, we performed simulations parameterized by the implemented rate of mass preventive and ring post-outbreak vaccinations. In the first step, $0,0.25,0.5$ or 0.75 of the population was randomly assigned the 'vaccinated' status (mass vaccination), one node among the remaining was either randomly selected (random initial infection) or the highest-degree node was selected (hub initial vaccination) to become an 'infected' node, and all of the remaining nodes were set as 'susceptible.' In each of the following step, all of the 'susceptible' nodes in contact with an 'infected' node were listed, and either $0,0.25$, 0.5 or 0.75 of them were assigned the 'vaccinated' status (ring vaccination). Each set of simulation was repeated five times. The parameters used for our simulation was $R_{0}$ $=0.5,1,2, \ldots, 64$, and the corresponding values for $T$, caclulated from the above mentioned relation between $R_{0}$ and $T$. (The values of $R_{0}$ differ widely according to diseases: influenza has 1.7, SARS has 1.2-3.6, smallpox has 4-10, and measles have $17[1]$.

\section{Acknowledgments}

This study was partially supported by the 'Special Coordination Funds for Promoting Science and Technology' from the Ministry of Education, Culture, Sports, Science and Technology.

\section{References}

1. Anderson, R.M. and R.M. May, Infectious diseases of humans : dynamics and control. Oxford science publications. 1991, Oxford ; New York: Oxford University Press. viii, $757 \mathrm{p}$.

2. Pastor-Satorras, R. and A. Vespignani, Epidemic spreading in scale-free networks. Physical Review Letters, 2001. 86(14): p. 3200-3203.

3. Liljeros, F., et al., The web of human sexual contacts. Nature, 2001. 411(6840): p. 907-8.

4. Amaral, L.A., et al., Classes of small-world networks. Proc Natl Acad Sci U S A, 2000. 97(21): p. 11149-52. 
5. Pastor-Satorras, R. and A. Vespignani, Immunization of complex networks. Physical Review E, 2002. 65: p. 036104.

6. Cohen, R., S. Havlin, and D. ben-Avraham, Efficient immunization strategies for computer networks and populations. Physical Review Letters, 2003. 91(24): p. 247901.

7. Meyers, L.A., et al., Network theory and SARS: predicting outbreak diversity. J Theor Biol, 2005. 232(1): p. 71-81. 